\title{
A simple model for Carnot heat engines
}

\author{
Jacques Arnaud \\ Mas Liron, F30440 Saint Martial, France \\ Laurent Chusseau \\ Institut d'Électronique du Sud, UMR No. 5214 au CNRS, Université Montpellier II, \\ F34095 Montpellier, France \\ Fabrice Philippe ${ }^{\text {a) }}$ \\ LIRMM, UMR No. 5506 au CNRS, 161 rue Ada, F34392 Montpellier, France
}

(Received 2 December 2008; accepted 23 September 2009)

\begin{abstract}
We discuss a model consisting of two reservoirs, each with $N$ possible ball locations, at heights $E_{h}$ and $E_{l}<E_{h}$ in a gravitational field. The two reservoirs contain $n_{h}$ and $n_{l}$ weight 1 balls. Empty locations are treated as weight 0 balls. The reservoirs are shaken so that all possible ball configurations are equally likely to occur. A cycle consists of exchanging a ball randomly chosen from the higher reservoir and a ball randomly chosen from the lower reservoir. We relate this system to a heat engine and show that the efficiency, which is defined as the ratio of the average work produced to the average energy lost by the higher reservoir, is $1-E_{l} / E_{h}$. When $n_{l}$ is comparable to $n_{h}$, the efficiency is found to coincide with the maximum efficiency $1-T_{l} / T_{h}$, where the temperatures $T_{l}$ and $T_{h}$ are defined from a simple expression for the entropy. We also discuss the evaluation of fluctuations and the history of the Carnot discovery. () 2010 American Association of Physics Teachers.
\end{abstract}

[DOI: $10.1119 / 1.3247983]$

\section{INTRODUCTION}

The purpose of this paper is to introduce several important thermodynamic concepts in a simple manner. The paper is self-contained and employs only elementary mathematics. The results are derived for a particular urn (or reservoir) model, which is related to the one introduced in 1907 by Ehrenfest. ${ }^{1-3}$ The model consists of two reservoirs, each with $N$ possible ball locations containing, respectively, $n_{l}$ and $n_{h}$ weight 1 balls. These balls are considered indistinguishable. This model is applicable to Otto heat engines (in which the working agent parameter stays fixed when in contact with either bath) with two-level atoms as the working agent. ${ }^{4,5}$

In Sec. II we describe our model and evaluate the average work produced, the average energy lost by the upper reservoir, and the efficiency. The general properties of heat engines are recalled in Sec. III. The relation between our urn model and the properties of heat engines is discussed in Sec. IV. The absolute temperatures $T_{h}$ and $T_{l}$ of the reservoirs are defined in the limit of large ball numbers. When $n_{l} \approx n_{h}$, the system efficiency and the average work reduce to the expression for the efficiency and average work given by Carnot for an ideal heat engine. The fluctuations in the work produced are evaluated in Appendix A. A discussion of the history of the Carnot discovery is given in Appendix B.

\section{EXCHANGE OF BALLS BETWEEN TWO RESERVOIRS}

We consider a system consisting of two reservoirs at heights $E_{l}$ and $E_{h}>E_{l}$ with respect to some lower reference level. There are $N$ possible ball locations in each reservoir, which we label as $1,2, \ldots, N$, as shown in Fig. 1. A single ball is allowed in any given location. The lower reservoir contains $n_{l}$ weight 1 balls, and the higher reservoir contains $n_{h}$ weight 1 balls. In Fig. 1 we show an example with $N=5$, $n_{h}=2$, and $n_{l}=1$. In the higher reservoir there are ten distin- guishable ball configurations, and in the lower reservoir there are five distinguishable configurations. The two reservoirs together have $5 \times 10=50$ distinguishable configurations, all of which correspond to the same energy. If we assume that the reservoirs are sufficiently shaken between exchange events, all possible ball configurations are equally likely to occur.

We consider ball displacements from the lower and higher reservoirs at a single location, say, the left-most location, labeled 1. We may draw all the possible configurations and evaluate by inspection quantities of interest such as the average work produced by the system. For example, the nine distinguishable configurations for $N=3, n_{h}=2$, and $n_{l}=1$ are shown in Fig. 2. The number below each configuration is the energy $E$ produced over a cycle, with $E \equiv E_{h}-E_{l}=1$. For example, consider the first position of the first configuration, and note that there is an empty location in the higher reservoir and a ball in the lower reservoir. In this case a cycle consists of transferring the lower-reservoir ball to the higher empty location. The system then delivers an energy equal to -1 , that is, the system absorbs an energy equal to 1 . The average energy produced for this configuration, the average work, is $\langle W\rangle=(-1+0+0+0+1+1+0+1+1) / 9=1 / 3$.

We now turn to an equivalent but more convenient picture involving the uniform probability of choosing a ball from a reservoir. It is then convenient to suppose that empty locations are occupied by weight 0 balls, which do not carry any energy when displaced. A cycle consists of exchanging two balls (of weight 0 or 1), one randomly chosen from the lower reservoir and one randomly chosen from the higher reservoir. The probability of choosing a weight 1 ball from the lower reservoir is $p_{l} \equiv n_{l} / N$, and the probability of picking up a weight 1 ball from the higher reservoir is $p_{h} \equiv n_{h} / N$. As we will discuss, the average energy produced is $\langle W\rangle=p_{h}-p_{l}$. For our example with $N=3, n_{h}=2$, and $n_{l}=1$, we have $p_{l}=1 / 3$, 


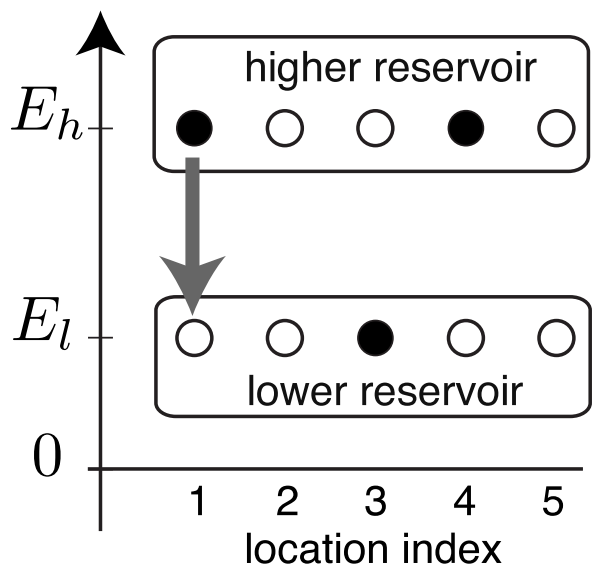

Fig. 1. Schematic representation of an engine that converts potential energy into work. Two reservoirs are located at heights $E_{l}$ and $E_{h} \geq E_{l}$, each with $N$ possible ball locations labeled $1,2,3, \ldots, N(N=5$ in the figure). The number of weight 1 balls (black circles) is $n_{l}$ in the lower reservoir and $n_{h}$ in the higher reservoir. Open circles represent weight 0 balls. For each reservoir, every ball configuration is equally likely to occur with the same frequency because the energies are the same. Balls may be transferred from one reservoir to the other in location 1 only. If there is a ball in the upper reservoir at that location and none in the lower one (as is the case in the figure), the ball is transferred from the upper reservoir to the lower one, thereby delivering energy. Conversely, if there is a ball in the lower reservoir and none in the upper one, the ball is transferred from the lower reservoir to the upper one, thereby absorbing energy.

$p_{h}=2 / 3$, and $\langle W\rangle=2 / 3-1 / 3=1 / 3$, which coincides with the previous result. The equivalence between the two methods is general.

The total energy of the system with $n$ weight 1 balls is $Q=n E$ (with respect to some arbitrary level). The letter $Q$ is employed anticipating a correspondence with thermodynamics. When a weight 1 ball is added to the reservoir at height $E$, the reservoir energy is incremented by $E$. If a ball is randomly chosen from the $N$ locations of a reservoir containing $n$ weight 1 balls, the probability that this ball has weight 1 is $n / N$. Hence, if the chosen ball is subsequently carried to a reservoir at height $E$, the latter reservoir's average energy is incremented by $\Delta Q=E n / N$. The word "average" will henceforth be omitted because only average values will be considered.

Consider two such reservoirs, one at height $E_{l}$ (lower reservoir) with $n_{l}$ weight 1 balls and the other at height $E_{h}$ (higher reservoir) with $n_{h}$ weight 1 balls. A cycle consists of exchanging two randomly chosen balls between the two reservoirs. The energies added to the lower and higher reservoirs are, respectively,

$$
\Delta Q_{l}=E_{l}\left(p_{h}-p_{l}\right), \quad \Delta Q_{h}=-E_{h}\left(p_{h}-p_{l}\right) .
$$

The work performed follows from conservation of energy

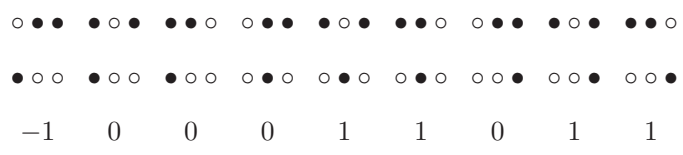

Fig. 2. The nine possible configurations for $N=3, n_{h}=2$, and $n_{l}=1$. The number below each configuration is the energy produced for one cycle.

$$
W=-\Delta Q_{l}-\Delta Q_{h}=\left(E_{h}-E_{l}\right)\left(p_{h}-p_{l}\right) .
$$

The engine efficiency, defined as the ratio of the work performed $W$ and the energy $-\Delta Q_{h}$ lost by the higher reservoir, is therefore

$$
\eta \equiv \frac{W}{-\Delta Q_{h}}=1-\frac{E_{l}}{E_{h}}
$$

In Sec. IV we show that when $p_{h} \approx p_{l}$, the efficiency given in Eq. (3) coincides with the Carnot efficiency, and the work given in Eq. (2) coincides with the expression given by Carnot.

After a cycle the number of weight 1 balls in a reservoir is changed by \pm 1 or 0 . The next cycle therefore operates with different values of $p_{l}$ and $p_{h}$. We will not consider the evolution of the work produced cycle after cycle. We are interested in a comparison with heat engines operating between two baths whose heat capacity is so large that their temperatures do not vary significantly. Likewise, in the present reservoir model, we may suppose that the number of balls in each reservoir is so large that their change after any number of cycles is insignificant.

\section{HEAT ENGINES}

In this section we recall some well known facts about heat engines (for a detailed discussion, see, for example, Ref. 6).

It is known empirically that when two bodies at different temperatures are placed in contact, they eventually reach the same temperature. This property may be interpreted by supposing that energy is transferred from the high-temperature body to the low-temperature body but that the converse never occurs. A similar observation can be made with two urns (see Fig. 1 with $E_{l}=E_{h}$ ). Suppose that the urns contain $N$ balls, each of which is either a weight 1 (black) or weight 0 (white) ball. If randomly chosen balls are repeatedly exchanged between the two urns, the ratios of the black and white balls will eventually become nearly the same in both urns independent of the initial conditions. For the analogy with thermal contact to hold, we need to define the temperature of an urn as a monotonically increasing function of the ratio of the number of black to white balls, as long as $n_{h}$ $<N / 2$.

It is also known empirically that when energy is transferred to a body, the body's temperature rises. We say that the body has received energy by heating. The fact that this energy cannot be converted back into usable energy is one form of the second law of thermodynamics. To obtain usable energy from heating, we need two bodies at different temperatures.

Let us now recall how usable energy may be retrieved from two baths at different temperatures. What we need is a "working agent," which may be any piece of material whose properties can be changed by varying a parameter. An example is a helium-filled cylinder with a piston of variable length $\epsilon$. Another example more relevant to this paper is a collection of two-level atoms. We suppose that the separation in energy $\epsilon$ of the two levels may be varied by, for example, the application of an electrical field. The external agent that causes the parameter $\epsilon$ to vary transfers energy. A typical closed cycle consists of putting the working agent with parameter $\epsilon_{1}$ in contact with the low-temperature bath and slowly varying $\epsilon$ to $\epsilon_{2}$. The working agent is then carried from the low-temperature bath to the high-temperature bath, 
while $\epsilon$ is slowly changed to $\epsilon_{3}$. The parameter is then changed to $\epsilon_{4}$. The working agent is finally returned to the low-temperature bath, and the parameter recovers its initial $\epsilon_{1}$ value. A closed cycle is thus defined by the nature of the working agent and four parameter values. When $\epsilon$ does not vary the cycle transfers energy from the high-temperature bath to the low-temperature bath and is analogous to a thermal contact.

For the Otto cycle, which describes an idealized form of the gasoline engine, ${ }^{7} \epsilon_{2}=\epsilon_{1}$ and $\epsilon_{4}=\epsilon_{3}$, meaning that the parameter does not vary when the working agent is in contact with either bath; $\epsilon$ varies only during the adiabatic (that is, slow and with thermal isolation) transitions from one bath to the other. This cycle may deliver or receive work for appropriate choices of the parameters. Usually it does not achieve the maximum (Carnot) efficiency, but it does so approximately when $\epsilon_{4} \approx \epsilon_{2}$. For the exact description of Carnot heat engines, see the generalization of the ball model in Ref. 8 .

\section{ENTROPY AND TEMPERATURE}

We consider a reservoir with $N$ locations and $n$ weight 1 balls and $N-n$ weight 0 balls. The number of ball configurations in the reservoir is $N ! / n !(N-n) !{ }^{9}$ We define the entropy as the logarithm of the number of configurations, the Boltzmann constant being set equal to unity, that is,

$$
S(n)=\ln \left(\frac{N !}{n !(N-n) !}\right) .
$$

Note that for large $n$,

$$
\begin{aligned}
S(n+1)-S(n)= & \ln \left(\frac{N !}{(n+1) !(N-n-1) !}\right) \\
& -\ln \left(\frac{N !}{n !(N-n) !}\right) \\
= & \ln \left(\frac{N-n}{n+1}\right) \approx \ln \left(\frac{N}{n}-1\right),
\end{aligned}
$$

The absolute temperature of a reservoir is defined as

$$
T(n)=\frac{Q(n+1)-Q(n)}{S(n+1)-S(n)} \approx \frac{E}{\ln \left(\frac{N}{n}-1\right)} .
$$

The entropy $S$ must be an extensive quantity. Because the number of configurations in two separate bodies is the product of the configurations and the logarithmic function has the property that $\ln (a b)=\ln (a)+\ln (b)$, the final approximate expression in Eq. (7) ensures that $T$ is an intensive quantity.

The cycle efficiency given in Eq. (3) may now be written in terms of the temperature as

$$
\eta=1-\frac{E_{l}}{E_{h}}=1-\frac{T_{l}}{T_{h}} \frac{\ln \left(p_{l}^{-1}-1\right)}{\ln \left(p_{h}^{-1}-1\right)} .
$$

When $p_{l} \approx p_{h}$, the last term in Eq. (8) drops out, the Carnot efficiency is obtained, and the work $W$ produced per cycle is very small. However, we may always add up the work contributions of any number of similar devices having the same reservoir temperatures (but possibly different values of $E$ and $n$ ) and achieve any specified amount of work at the Carnot efficiency.
The ball exchange we have discussed changes the reservoir entropies by \pm 1 or 0 . Because these events are independent, the change in the entropy of the lower reservoir is

$$
\begin{aligned}
\Delta S_{l}= & p_{h}\left(1-p_{l}\right)\left[S\left(n_{l}+1\right)-S\left(n_{l}\right)\right] \\
& +p_{l}\left(1-p_{h}\right)\left[S\left(n_{l}-1\right)-S\left(n_{l}\right)\right] .
\end{aligned}
$$

From Eq. (6) we obtain

$$
\Delta S_{l}=\left(p_{h}-p_{l}\right) \ln \left(p_{l}^{-1}-1\right) \text {. }
$$

The change in the entropy of the higher reservoir is obtained by exchanging the $h$ and $l$ labels in Eq. (10), that is

$$
\Delta S_{h}=-\left(p_{h}-p_{l}\right) \ln \left(p_{h}^{-1}-1\right) .
$$

We thus find that for $p_{l} \approx p_{h}, \Delta S_{l} \approx-\Delta S_{h}$ so that there is no net entropy produced. Entropy is just carried from the higher reservoir to the lower one. The Carnot expression for the work may thus be written as

$$
\begin{aligned}
W=\left(T_{h}-T_{l}\right) \Delta S_{l} & \approx\left(\frac{E_{h}}{\ln \left(p_{h}^{-1}-1\right)}-\frac{E_{l}}{\ln \left(p_{l}^{-1}-1\right)}\right) \Delta S_{l} \\
& \approx\left(E_{h}-E_{l}\right)\left(p_{h}-p_{l}\right),
\end{aligned}
$$

so that the Carnot expression for $W$ coincides with the expression for the work performed per cycle evaluated. More precisely, the ratio of the total entropy produced $\Delta S_{l}+\Delta S_{h}$ to the work produced $W$ goes to zero as $p_{h} \rightarrow p_{l}$ [see Eq. (A4)].

Equation (13) implies that the engine delivers work only if $E_{h}-E_{l}$ and $p_{h}-p_{l}$ are both positive or both negative. Because $E_{h}>E_{l}$, we must have $p_{h}>p_{l}$. From Eq. (7) and that the logarithm is a monotonically increasing function of its argument, we find that work may be produced only if $T_{h}>T_{l}$.

Conventional heat engines operate with two large baths, or reservoirs, one hot and one cold. Because these baths are not infinite in size, the temperature of the hot bath decreases, and the temperature of the cold bath increases and eventually no work is produced. The same situation occurs in our model. After a very large number of cycles, the values of $p_{h}$ and $p_{l}$ coincide, and work is no longer produced. Because the reservoir temperatures do not equalize, we may say that the system has reached a state of equilibrium but not a state of thermal equilibrium. This kind of equilibrium is not a peculiarity of our model but a general property of some heat engines.

\section{ACKNOWLEDGMENTS}

The authors thank the anonymous referees for careful reading and useful comments.

\section{APPENDIX A: FLUCTUATIONS}

We obtain in the following the fluctuations in the work produced and the high-temperature reservoir energy loss. Recall that a cycle consists of simultaneously exchanging a ball from the higher reservoir with $n_{h}$ weight 1 balls and $N-n_{h}$ weight 0 balls and a ball from the lower reservoir with $n_{l}$ weight 1 balls and $N-n_{l}$ weight 0 balls.

We saw in the main text that the average work produced per cycle is $\langle W\rangle=E\left(p_{h}-p_{l}\right)$. We now evaluate $\left\langle W^{2}\right\rangle$. The probability that a weight 1 ball falls and none is raised is $p_{h}\left(1-p_{l}\right)$. If this event occurs, the work performed squared is equal to $E^{2}$. Conversely, the probability that a weight 1 ball 
is raised and none falls is $p_{l}\left(1-p_{h}\right)$. If this event occurs, the work performed squared is again equal to $E^{2}$. Because the two other cases produce no work, it follows that $\left\langle W^{2}\right\rangle$ $=E^{2}\left[p_{h}\left(1-p_{l}\right)+p_{l}\left(1-p_{h}\right)\right]$. Therefore, the variance of the work produced is

$$
\begin{aligned}
\left\langle W^{2}\right\rangle-\langle W\rangle^{2} & =E^{2}\left[p_{h}\left(1-p_{l}\right)+p_{l}\left(1-p_{h}\right)-\left(p_{h}-p_{l}\right)^{2}\right] \\
& =E^{2}\left[p_{h}\left(1-p_{h}\right)+p_{l}\left(1-p_{l}\right)\right] .
\end{aligned}
$$

For $p_{h} \approx p_{l}$ we have $\left\langle W^{2}\right\rangle-\langle W\rangle^{2} \approx 2 E^{2} p_{l}\left(1-p_{l}\right)$.

We now consider the total entropy produced $\Delta S \equiv \Delta S_{l}$ $+\Delta S_{h}$. When a weight 1 ball is transferred from the high to the lower reservoir and none from the low to the higher reservoir, an event that occurs with probability $p_{h}\left(1-p_{l}\right)$, the change in $S_{l}$ according to Eq. (6) is $\Delta S\left(n_{l}+1\right)-\Delta S\left(n_{l}\right)$ $=\ln \left(p_{l}^{-1}-1\right)$, and the change in $S_{h}$ is $\Delta S\left(n_{h}-1\right)-\Delta S\left(n_{h}\right)=$ $-\ln \left(p_{h}^{-1}-1\right)$. It follows that the change in the total entropy is $\ln \left(\left(p_{l}^{-1}-1\right) /\left(p_{h}^{-1}-1\right)\right)$ with probability $p_{h}\left(1-p_{l}\right)$. When a ball is transferred from the low to the higher reservoir and none from the high to the lower reservoir, an event that occurs with probability $p_{l}\left(1-p_{h}\right)$, the change in $S_{l}$ is, according to Eq. (6), $\Delta S\left(n_{l}-1\right)-\Delta S\left(n_{l}\right)=-\ln \left(p_{l}^{-1}-1\right)$, and the change in $S_{h}$ is $\Delta S\left(n_{h}+1\right)-\Delta S\left(n_{h}\right)=\ln \left(p_{h}^{-1}-1\right)$. It follows that the change in the total entropy is $\ln \left(\left(p_{h}^{-1}-1\right) /\left(p_{l}^{-1}-1\right)\right)$ with probability $p_{l}\left(1-p_{h}\right)$.

The average change in the total entropy is therefore

$$
\begin{aligned}
\langle\Delta S\rangle & =p_{h}\left(1-p_{l}\right) \ln \left(\frac{p_{l}^{-1}-1}{p_{h}^{-1}-1}\right)+p_{l}\left(1-p_{h}\right) \ln \left(\frac{p_{h}^{-1}-1}{p_{l}^{-1}-1}\right) \\
& =\left(p_{h}-p_{l}\right) \ln \left(\frac{p_{l}^{-1}-1}{p_{h}^{-1}-1}\right) \geq 0 .
\end{aligned}
$$

When $p_{h} \approx p_{l}, \Delta S \approx 0$, and the system becomes reversible and achieves the highest efficiency. Note that the entropy change is non-negative for both a heat engine $\left(p_{h}>p_{l}\right)$ and a heat pump $\left(p_{l}>p_{h}\right)$. More precisely, to first order in $\delta \equiv p_{h}$ $-p_{l}$ we have $\ln \left[\left(p_{l}^{-1}-1\right) /\left(1 / p_{h}^{-1}-1\right)\right] \approx \delta /\left[p_{l}\left(1-p_{l}\right)\right]$, and thus

$$
\langle\Delta S\rangle \approx \frac{\delta^{2}}{p_{l}\left(1-p_{l}\right)} .
$$

This result is reasonable because the entropy is proportional to $\delta^{2}$, and the work produced is proportional to $\delta$ so that for small $\delta$, near reversibility does not imply vanishing work.

We also obtain

$$
\begin{aligned}
\left\langle(\Delta S)^{2}\right\rangle= & p_{h}\left(1-p_{l}\right)\left[\ln \left(\frac{p_{l}^{-1}-1}{p_{h}^{-1}-1}\right)\right]^{2} \\
& +p_{l}\left(1-p_{h}\right)\left[\ln \left(\frac{p_{h}^{-1}-1}{p_{l}^{-1}-1}\right)\right]^{2} \\
= & \left(p_{h}+p_{l}-2 p_{l} p_{h}\right)\left[\ln \left(\frac{p_{l}^{-1}-1}{p_{h}^{-1}-1}\right)\right]^{2},
\end{aligned}
$$

and the variance is

$$
\begin{aligned}
\left\langle(\Delta S)^{2}\right\rangle-\langle\Delta S\rangle^{2}= & \left(p_{h}+p_{l}-2 p_{l} p_{h}\right. \\
& \left.-\left(p_{h}-p_{l}\right)^{2}\right)\left[\ln \left(\frac{p_{l}^{-1}-1}{p_{h}^{-1}-1}\right)\right]^{2} \\
= & {\left[p_{h}\left(1-p_{h}\right)+p_{l}\left(1-p_{l}\right)\right] } \\
& \times\left[\ln \left(\frac{p_{l}^{-1}-1}{p_{h}^{-1}-1}\right)\right]^{2},
\end{aligned}
$$

which vanishes, as well as the average entropy produced, when $p_{h} \approx p_{l}$. To first order in $\delta$ we have

$$
\left\langle(\Delta S)^{2}\right\rangle-\langle\Delta S\rangle^{2} \approx 2\langle\Delta S\rangle,
$$

a remarkably simple result. This result was given in Ref. 10 and in previous work. ${ }^{11,12}$ This agreement shows that the properties of our model are generally applicable to heat engines.

\section{APPENDIX B: BRIEF HISTORY OF CARNOT'S DISCOVERIES}

The motivation for introducing the following account of Carnot discoveries is that they remain insufficiently appreciated.

The Carnot theory, which appeared in a book in 1824 and in unpublished notes, established both the first and the second laws of thermodynamics. ${ }^{13}$ This fact has been pointed out by several authors who have looked carefully at what Carnot actually wrote, clarifying the terminology employed, updating the system of units, and correcting minor errors in the experimental data. We translate from Kastler's paper: ${ }^{14}$ "Had Sadi Carnot lived longer [...], he would be considered today not only as the author of the Carnot principle (called by Clausius the second principle of thermodynamics) but also as the author of the first principle of that science." The book by Brodiansky ${ }^{13}$ lists ten major achievements of Carnot, including that "Carnot is the first to formulate the second principle of thermodynamics" and "He was among the first to formulate strictly the law of equivalence between heat and work and the first to calculate with sufficient accuracy its numerical value."

One reason for the lack of appreciation of Carnot's contribution is that it appeared in print decades after his early death. A second reason is that his work was popularized by Clapeyron in a partly erroneous manner. A third reason is the unfortunate use by Carnot of the word "calorique" to designate what Clausius later called "entropy." The word calorique had been formerly employed by Lavoisier to designate some hypothetical heat substance. Clausius and Lord Kelvin, although highly appreciative of Carnot's work, missed part of his contribution because Carnot's notes were not available to them. La Mer expressed himself forcefully as follows: "Unless the view-point that the Carnot theory is accurate is adopted, one is placed in the position of maintaining that Carnot succeeded in demonstrating some of the most fundamental and profound principles of physical science by the most masterly display of scientific double-talk that has ever been perpetrated upon the scientific world. This view is untenable." ${ }^{15}$ Much clarification is due to Hoyer. ${ }^{16}$ The historian of science Fox has written, "Until recently there were very few studies concerning [the physics of Carnot reflections]. Thanks to the work of Hoyer, we now have papers on the logical implications of the Carnot theory, and its analogy 
with modern thermodynamics [...]. It is not at all obvious to understand how Carnot [discovered the mechanical equivalent of heat]. Hoyer examined this question in two important papers. His articles provide complete references to earlier attempts $[\ldots]$. He explains the exactness of Carnot calculation (which is even more striking if one uses modern values for the specific heats) by noticing that the Carnot theory is entirely accurate."

As far as the first law of thermodynamics is concerned, we quote Carnot: ${ }^{17}$ "Heat is nothing but motive power, or rather another form of motion. Wherever motive power is destroyed, heat is generated in precise proportion to the quantity of motive power destroyed; conversely, wherever heat is destroyed, motive power is generated." (Carnot calculated that $1 \mathrm{cal}$ is equivalent to $3.27 \mathrm{~J}$ instead of the present-day value of $4.18 \mathrm{~J}$.)

The key fact related to the second law is that engine efficiencies reach their maximum value when they are reversible. Carnot reached this conclusion by considering that energy cannot be obtained for free. He therefore looked for energy transfers that could work in a reversed manner. Slow processes are reversible, with the exception of thermal contacts. Because there is some confusion in the literature concerning the significance of the Carnot contribution to the second law of thermodynamics, we quote Zemansky and Dittman: "Carnot used chaleur when referring to heat in general, but when referring to the motive power of fire that is brought about when heat enters an engine at high temperature and leaves at low temperature, he uses the expression chute de calorique, never chute de chaleur [...]. Carnot had in the back of his mind the concept of entropy, for which he reserved the term calorique."

\footnotetext{
a) Also at Université Montpellier 3, Route de Mende, F34199 Montpellier, France
}

${ }^{1}$ P. Ehrenfest and T. Ehrenfest, "Ueber zwei bekannte Eingewände gegen das Boltzmannsche H-Theorem," Z. Phys. 8, 311-314 (1907).

${ }^{2}$ J. Güémez, S. Velasco, and A. Calvo Hernández, "A generalization of the Ehrenfest urn model,” Am. J. Phys. 57, 828-834 (1989).

${ }^{3}$ J. Tobochnik and H. Gould, "Teaching statistical physics by thinking about models and algorithms," Am. J. Phys. 76, 353-359 (2008).

${ }^{4}$ Classical heat engines may be considered as special cases of quantum heat engines.

${ }^{5}$ H. T. Quan, "Quantum thermodynamic cycles and quantum heat engines (II)," Phys. Rev. E 79, 041129-1-10 (2009).

${ }^{6}$ M. Zemansky and R. Dittman, Heat and Thermodynamics (McGraw-Hill, New York, 1997).

${ }^{7}$ H. B. Callen, Thermodynamics and an Introduction to Thermostatistics (Wiley, New York, 1985).

${ }^{8}$ J. Arnaud, L. Chusseau, and F. Philippe, "Mechanical equivalent of quantum heat engines," Phys. Rev. E 77, 061102-1-6 (2008).

${ }^{9}$ If the balls could be distinguished from one another, the number of configurations would be $N(N-1) \ldots(N-n+1)$, but this number must be divided by $n$ ! to account for the fact that the exchange of balls is irrelevant.

${ }^{10}$ J. Arnaud, L. Chusseau, and F. Philippe, "Carnot cycle for an oscillator," Eur. J. Phys. 23, 489-500 (2002).

${ }^{11}$ C. Jarzynski, "Nonequilibrium equality for free energy differences," Phys. Rev. Lett. 78, 2690-2693 (1997).

${ }^{12}$ G. E. Crooks, "Entropy production fluctuation theorem and the nonequilibrium work relation for free energy differences," Phys. Rev. E 60, 2721-2726 (1999).

${ }^{13}$ V. M. Brodiansky, Sadi Carnot (Presses Universitaires de Perpignan, Perpignan, 2006), translated from Russian to French.

${ }^{14}$ A. Kastler, Sadi Carnot et l'Essor de la Thermodynamique (Editions du CNRS, Paris, 1976), p. 195.

${ }^{15}$ V. K. La Mer, "Some current misinterpretations of N. L. Sadi Carnot's memoir and cycle," Am. J. Phys. 22, 20-26 (1954).

${ }^{16}$ U. Hoyer, "How did Carnot calculate the mechanical equivalent of heat?," Centaurus 19(3), 207-219 (1975).

${ }^{17}$ N. S. Carnot, Réflexions sur la Puissance Motrice du Feu et sur Les Machines Propres à Développer cette Puissance (Bachelier, Paris, 1824); translated to English by R. B. Thurston from the original French of N.L.S. Carnot and accompanied by an account of Carnot's theory by Sir William Thomson (Lord Kelvin) in Reflections on the Motive Power of Heat (John Wiley and Sons, New York, 1897). 